\title{
Are Self-regarding Subjects More Rational?
}

\author{
Benito Arruñada, Marco Casari and Francesca Pancotto*
}

January 16, 2012

\begin{abstract}
Through an experiment, we investigate how the level of rationality relates to concerns for equality and efficiency. Subjects perform dictator games and a guessing game. More rational subjects are not more frequently of the selfregarding type. When performing a comparison within the same degree of rationality, self-regarding subjects show more strategic sophistication than other subjects.
\end{abstract}

\section{JEL codes: C91 C92 D63}

\section{Keywords: steps of reasoning, other-regarding preferences}

\footnotetext{
*Corresponding author: Pancotto, Universita' di Modena and Reggio Emilia, Department of Comunication and Economics, Reggio Emilia, email: francesca.pancotto@unimore.it. Arruñada: Pompeu Fabra University and Barcelona GSE, email: benito.arrunada@upf.edu; Casari: Universita' di Bologna, Department of Economics, email: marco.casari@unibo.it. We thank Stefania Bortolotti for useful comments and Jingjing Zhang and Sukanya Chaudury for research assistance. Usual disclaimers apply. Funding for this research was provided by Purdue University, the Spanish Ministry of Science and Innovation, through grants SEJ2005-03871/ECON and ECO2011-29445, and the European Commission through Project CIT3513420 .
} 
Modeling preferences of economic agents is at the center of a long lasting debate about human behavior. There exists evidence that people respond to motivations that go beyond the maximization of personal material payoffs (Fehr and Schmidt, 1999; Bolton and Ockenfels, 2000) and exhibit bounded rationality when taking decisions with economic content (Selten, 1998). In an experiment we study how different distributional preferences relate to measures of bounded rationality. We employed three player dictator games to elicit concerns for efficiency and for equality (Charness and Rabin, 2002; Engelmann and Strobel, 2004; Cox and Sadiraj, 2010) and a guessing game to evaluate their depth of reasoning (Nagel, 1995; Grosskopf and Nagel, 2008).

Dictator games are simple and not affected by any strategic consideration. They can discriminate precisely between different distributional motives and can help in disentangling types of behavior related to other-regarding preferences. On the other hand, the guessing game is well-suited to investigate strategic thinking and evaluate the degree of (bounded) rationality of subjects. According to Ho et al. (1998), in a guessing game "[t]he presence of a limited number of steps of iterated dominance could be taken as a sharp measure of the degree of bounded (mutual) rationality." The guessing game is a constant sum game, thus concerns for efficiency do not affect behavior; moreover the equilibrium strategy is the same for self-regarding and for other-regarding preferences, hence providing a measure of strategic rationality that is independent from social concerns.

The paper is structured as follows. Section 1 describes the experimental design, Section 2 reports the results. Section 3 concludes.

\section{Experimental design}

Experiments were run at Purdue University. Overall 195 undergraduate students participated in 16 sessions. Each subject participated in only one of the sessions. ${ }^{1}$

At the beginning of each session, we elicited the preferences of all subjects with respect to concern for equality and efficiency in a static context, along the lines of Engelmann and Strobel (2004). We presented two tables to each subject (Table 1). Each table proposed a choice between alternative allocations of money among a group of three persons. Person 2 was the dictator in the group. The experiment elicited each participant's choice as if she was the dictator. In order to have more observations, we asked all subjects to choose, before knowing if their decision mattered or not. At the end of the session, roles were

\footnotetext{
${ }^{1} \mathrm{~A}$ session included also other tasks that were later explained to participants. The complete description is in Arrunada and Casari (2010). Here we analyze the same data studied in the cited paper.
} 
assigned randomly and the decisions of persons 1 and 3 had no impact on the outcome. Participants were instructed to choose among the available options as if they knew they were the dictator (Person 2). Choices were written on a personal card. The experimenter collected the cards, shuffled them, randomly formed groups, and randomly assigned roles. For each group, the card of the subject in role 2 (Person 2) was used to define the earnings in the group. The choices written on the cards of persons 1 and 3 were ignored. Half of the groups were paid according to choices made in the first table (Panel 1: A, B, C) and the other half to choices made in the second table (Panel 2: D, E, F). ${ }^{2}$ Subjects learned the results at the end of the session. Predictions for common models of other-regarding agents - such as Bolton and Ockenfels (2000) and Fehr and Schmidt (1999) - are in Table 1.

After this task we ran a one-shot guessing game in which all subjects had to write a real number between 0 and 100 on their personal decision cards. They were informed that groups of three would be randomly formed, and then a target number for each group would be computed by taking two thirds $(p=2 / 3)$ of the group average. Within each group, the subject closest to her target number received 6 points, which were evenly split in case of a tie.

After everybody made a choice, the experimenter collected all decision cards and wrote the results for both tasks on the cards, and returned to the subjects at the end of the session. The prize was split equally and individual earnings were 2 points. A point was worth USD 0.45. Earnings were paid privately at the end of a session. Regarding predictions, the best choices for a rational, self-regarded agent in the earlier task are $\mathrm{C}$ and $\mathrm{F}$. In the guessing game, the Nash equilibrium is to choose the number 0.

\section{Results}

We present raw data about choices in the dictator game and in the guessing game separately. In Table 1 one can see that $48 \%$ of subjects show a strong preference for equality (A in Panel 1) and 51\% show a strong preference for efficiency (D in Panel 2).

The frequency of choices in the guessing game is instead in Figure 1. Mean and median values are in line with those reported in Nagel (1995) for the one-shot guessing game with $\mathrm{p}=2 / 3$. A way to analyze the data of the guessing games is through the well-known Iterated Best Reply model (IBR from now on) (Nagel, 1995; Camerer et al., 2004). In this

\footnotetext{
${ }^{2}$ Participants decided under a 'veil of ignorance' because their choice had 1/3 probability of being enacted, thus inherently different from Rawls, where the pivotal person taking the allocation decision may end up as person 1, 2, or 3. Here the pivotal person is always person 2 .
} 
model each subject has a uniform prior over other players' choices and applies one level of reasoning deeper than what she believes that the other players will do. The IBR model can be specified under degenerate or non-degenerate beliefs about the other players. Under degenerate belief everyone assumes that all the other players are at the same specific level of reasoning. Under non-degenerate beliefs "... a subject gives positive probabilities to the other players being at more than on level of reasoning" (Ho et al., 1998). In Figure 1, next to the horizontal axis, we report the reference values to classify choices according IBR model and Iterated Dominance (ID) model as well. In ID model (Ochs, 1995), rational agents will reach the Nash equilibrium outcome after a series of steps: values larger than $100 \mathrm{p}$ - dominated strategies - are initially eliminated (with p being $2 / 3$ ), then values larger than $100 p^{2}$, and so on and so forth up to the Nash equilibrium in which everyone chooses 0. In our analysis, we focus on the IBR model, largely recognized as more apt to capture and explain behavior in the guessing game (Nagel, 1995) ${ }^{3}$.

For simplicity, we consider an IBR model with degenerate beliefs: In this case Step 0 players choose randomly, Step 1 players choose the best reply to 50, which is the mean value considering a uniform distribution of possible values with support $[0,100]$. Step 2 players believe that all other players apply Step 1 reasoning, hence Step 2 players best reply to a value of $50 p^{2}$, Step 3 best reply to $50 p^{3}$, and so on and so forth ${ }^{4}$.

We look at a possible relationship between bounded rationality and concerns for equality and efficiency. To this end, we classify subjects according to the steps of reasonings in the IBR model and then graph the share of subjects who chose a specific earnings allocation by step of reasoning, in Figure 2.

Consider for a moment the left panel, which reports choices concerning equality. Line A illustrates the fraction of subjects who preferred strict equality $(\mathrm{A})$, among those that the IBR model classifies as Step 0, Step 1, etc. Similarly, line C illustrates the fraction of subjects who preferred to maximize personal earnings. One can see that the shares of choices for the self-regarding option do not increase for higher steps of reasoning, when we compare it with the choices for equality. When dividing subjects into steps of reasoning, a similar share of subjects in each step chose the self-regarding option over the equality option.

On the contrary, in the right panel, we report a comparison of efficiency (D), versus self-regard (F). The right panel of Figure 2 suggests that the fraction of subjects that have a higher concern for efficiency increases for higher steps of reasoning.

\footnotetext{
${ }^{3}$ See Coricelli and Nagel (2009) for references.

${ }^{4}$ In the case of non-degenerate beliefs, the initial reference point is a realization from a uniform distribution function with mean value 50 .
} 
Result 1: The share of subjects who prefer equality over self-regard is similar across steps of reasoning. The share of subjects who prefer efficiency over self-regard is larger for higher steps of reasoning.

Table 3 reports the marginal effects of a series of probit regressions on individual choices for $\mathrm{A}, \mathrm{C}, \mathrm{D}$, and $\mathrm{F}$, respectively. In the first regression, the dependent variable takes value 1 when the subject chose $\mathrm{A}$ in the dictator game of Table 1 , Panel 1, and 0 otherwise. The regressors are a list of dummy variables that code the number of steps of reasoning according to the IBR model.

The regressions show that there is no significant relationship between the distributional choices with respect to equality concerns and the number of steps of reasoning. On the contrary, there is a significant relationship between the distributional choices with respect to efficiency and the number of steps of reasoning. In particular, those subjects who performed more than two steps of reasoning are less likely to have made the self-regarding choice $\mathrm{F}^{5}$

Going beyond the composition of the population characterizing each step of reasoning, we investigate the distribution of the values that these same subjects expressed across the dimension of preferences on income distribution. In Table 2 we compared the average numerical guesses of those who cared most for equality $(\mathrm{A})$ with the average guess of those who chose the self-regarding option (C), in each step of reasoning. A similar statistic was computed for those who chose $\mathrm{D}$ versus $\mathrm{F}$. The mean guess related to the choice of $\mathrm{A}$ is larger than the value for the choice of $\mathrm{C}$. Moreover, in all steps, the mean guess related to the choice of $\mathrm{D}$ is larger than the value for the choice of $\mathrm{F}$. A series of WilcoxonMann-Whitney and Kolmogorov-Smirnov tests ${ }^{6}$ compared the distributions of the guesses of subjects who chose A vs C and D vs F, classified according to the IBR model.

Both tests accept the hypothesis that the distribution of the values of $\mathrm{A}$ are significantly larger than $\mathrm{C}$ for higher steps of reasoning - for Step 2 and Higher Steps; the same holds for $\mathrm{D}$ versus $\mathrm{F}$ for Higher Steps (Table 2) .

Result 2: When performing step by step comparisons, the mean guess of subjects who prefer strict equality is significantly larger than the mean guess of those who favors personal

\footnotetext{
${ }^{5}$ In addition we performed similar regressions including among the regressors also the value of the guess, to inspect the relation between the value of guess and self-regarding choice. Results are reported in the Appendix. Regressions where the value of the guessing game is the only regression report no significant correlation. When both the dummies and the value of the guessing game are introduced, results are show how self-regarding subjects who have a high level of rationality exhibit much lower values of the guessing game - a value much closer to the Nash equilibrium - with respect to those preferring equality or efficiency.

${ }^{6}$ The p-values of the KS test involve a bootstrap procedure with 10000 repetitions.
} 
gains. Moreover, the mean guess of subjects who prefers maximum efficiency is larger than the mean guess of those who favors personal gains.

This result appears in Figure 3. The left panel depicts the mean guess of the participants who chose A normalized by the median calculated for the relative step of reasoning (solid line), compared to the mean value of subjects who chose $\mathrm{C}$ again normalized by the median calculated for the relative step of reasoning (dotted line). The normalization for the median facilitates visual comparability among observations across different steps of reasoning. The right panel of Figure 3, illustrates the choices for D versus F.

\section{Conclusions}

We have a proxy for strategic rationality and proxies for other-regarding preferences that are independent one from another and 'clean'. They are independent because they come from two separate games. They are clean because, on the one hand, the depth of strategic reasoning emerges from behavior in the guessing game, where the other-regarding orientation plays no role (Ho et al., 1998); on the other hand, three-players dictator games allow to classify subjects into types according to their concern for equality and efficiency and the game has no room for strategic motivations.

We can summarize the results into five points. First, are more rational subjects more frequently of the self-regarding type? The answer is no. The overall evidence shows that subjects engaging in higher steps of reasoning on average are as many exhibiting concerns for equality and efficiency as the other subjects. Second, the answer is linked also to how we identify self-regard in practice, whether we contrast it with a concern for efficiency or, instead, for equality. We report that the relation between other-regarding preferences and strategic rationality is stronger with respect to concerns for efficiency than to concerns for equality. Third, subjects engaging in higher steps of reasoning exhibit on average more concerns for efficiency than other subjects. Fourth, subjects engaging in higher steps of reasoning exhibit similar concerns for equality than other subjects. Fifth, we can also reverse the question into the following: are more rational subjects more self-regarding?

The answer is yes. When performing a comparison within the same step of reasoning, self-regarding subjects show more strategic sophistication than other subjects. 


\section{References}

Arrunada, B. and M. Casari (2010). How enforcement institutions affect markets. University of Bologna, WP DSE n. 616.

Bolton, G. E. and A. Ockenfels (2000). Erc: A theory of equity, reciprocity, and competition. The American Economic Review 90(1), 166-193.

Camerer, C., T. H. Ho, and J. K. Chong (2004). A cognitive hierarchy model of games. The Quarterly Journal of Economics 119(3), 861 - 898.

Charness, G. and M. Rabin (2002). Understanding social preferences with simple tests. The Quarterly Journal of Economics 117(3), pp. 817-869.

Coricelli, G. and R. Nagel (2009). Neural correlates of depth of strategic reasoning in medial prefrontal cortex. Proceedings of the National Academy of Science 106(23), 37 46.

Cox, J. C. and V. Sadiraj (2010). Direct tests of individual preferences for efficiency and equity. Economic Inquiry, forthcoming.

Engelmann, D. and M. Strobel (2004). Inequality aversion, efficiency, and maximin preferences in simple distribution experiments. The American Economic Review 94(4), 857-869.

Fehr, E. and K. M. Schmidt (1999). A theory of fairness, competition, and cooperation. The Quarterly Journal of Economics 114(3), pp. 817-868.

Grosskopf, B. and R. Nagel (2008). The two-person beauty contest. Games and Economic Behavior 62(1), 93 - 99 .

Ho, T., C. Camerer, and K. Weigelt (1998). Iterated dominance and iterated best response in experimental 'p-beauty contests'. The American Economic Review 88(4), 947 - 969.

Nagel, R. (1995). Unraveling in guessing games: An experimental study. American Economic Review 85(5), 1313-26.

Ochs, J. (1995). Games with unique, mixed strategy equilibria: An experimental study. Games and Economic Behavior 10(1), 202-217.

Selten, R. (1998). Features of experimentally observed bounded rationality. European Economic Review 42(3-5), 413-436. 


\section{Tables and Figures}

Table 1: Three person dictator games.

\begin{tabular}{|c|c|c|c|c|c|c|}
\hline & \multicolumn{3}{|c|}{ Panel 1 - Equality } & \multicolumn{3}{|c|}{ Panel 2 - Efficiency } \\
\hline & A & B & $\mathrm{C}$ & $\mathrm{D}$ & $\mathrm{E}$ & $\mathrm{F}$ \\
\hline Person 1 & 8 & 11 & 12 & 20.5 & 12 & 7.5 \\
\hline Person 2 (dictator) & 8 & 8.5 & 9 & 6.5 & 7 & 7.5 \\
\hline Person 3 & 8 & 4.5 & 3 & 5 & 5 & 5 \\
\hline Total & 24 & 24 & 24 & 32 & 24 & 20 \\
\hline Average earning of 1,3 & 8 & 7.75 & 7.5 & 12.75 & 8.5 & 6.25 \\
\hline Experimental Choices & \multicolumn{3}{|c|}{$\mathrm{N}=195$} & \multicolumn{3}{|c|}{$\mathrm{N}=195$} \\
\hline $\mathrm{N}$ of subjects & 94 & 27 & 74 & 100 & 32 & 63 \\
\hline Percentage & 48.2 & 13.8 & 37.9 & 51.3 & 16.4 & 32.3 \\
\hline Mean guess & 42.7 & 39.0 & 39.7 & 39.6 & 44.6 & 41.6 \\
\hline Median guess & 38.0 & 36.0 & 37.5 & 35.5 & 41.6 & 42.0 \\
\hline \multicolumn{7}{|l|}{ Predictions } \\
\hline Self-regarding & & & $\mathrm{C}$ & & & $\mathrm{F}$ \\
\hline Efficiency & - & - & - & $\mathrm{D}$ & & \\
\hline Bolton and Ockenfels & A & & & & & $\mathrm{F}$ \\
\hline Fehr and Schmidt & A & & & & & $\mathrm{F}$ \\
\hline Maximin & A & & & - & - & - \\
\hline
\end{tabular}


Table 2: Mean Guesses within steps of reasoning and distributional choices.

\begin{tabular}{llccccc}
\hline Equality & & \multicolumn{3}{c}{ Mean guess } & \multicolumn{2}{c}{ Equality vs Self-regard } \\
& & Equality & & Self-regard & \multicolumn{2}{c}{$A>C$} \\
Steps & guess ranges & A & B & C & MW & KS \\
\hline Step 0 & $(50,100)$ & 71.24 & 76.60 & 65.73 & 0.19 & 0.15 \\
Step 1 & $(33.33,50]$ & 41.73 & 44.03 & 41.35 & 0.34 & 0.23 \\
Step 2 & $(22.22,33.33]$ & 29.72 & 28.61 & 27.95 & $0.03^{* *}$ & $0.08^{*}$ \\
Higher Steps & $(0,22.22]$ & 15.03 & 14.32 & 8.64 & $0.09^{*}$ & $0.02^{* *}$ \\
\hline Efficiency & & \multicolumn{3}{c}{ Mean guess } & Efficiency vs Self-regard \\
& & Efficiency & \multicolumn{7}{c}{ Self-regard } & & $D>F$ \\
Steps & guess ranges & D & E & F & MW & KS \\
\hline Step 0 & $(50,100)$ & 72.08 & 71.21 & 65.64 & 0.11 & 0.15 \\
Step 1 & $(33.33,50]$ & 42.07 & 41.48 & 41.78 & 0.35 & 0.52 \\
Step 2 & $(22.22,33.33]$ & 29.18 & 29.36 & 27.84 & 0.15 & 0.30 \\
Higher Steps & $(0,22.22]$ & 13.81 & 15.67 & 8.25 & $0.06^{*}$ & $0.06^{*}$ \\
\hline
\end{tabular}

The mean value of the guessing game subdivided per steps of reasoning and choices in the distribution games. MW test and KS performed on guesses separately for levels of reasoning. Significance: *** $99 \%$, $* * 95 \%, * 90 \%$. 
Table 3: Steps of reasoning and concern for efficiency and equality.

\begin{tabular}{|c|c|c|c|c|}
\hline & \multicolumn{4}{|c|}{ Dependent Variable } \\
\hline & A (equality) & C (self-regard) & D(efficiency) & $\mathrm{F}$ (self-regard) \\
\hline \multirow[t]{2}{*}{ Step 0} & 0.13 & -0.08 & 0.01 & -0.08 \\
\hline & $(0.09)$ & $(0.08)$ & $(0.09)$ & $(0.07)$ \\
\hline \multirow[t]{2}{*}{ Step 2} & 0.04 & -0.01 & 0.12 & -0.15 \\
\hline & $(0.10)$ & $(0.09)$ & $(0.10)$ & $(0.08)$ \\
\hline \multirow[t]{2}{*}{ Higher Steps } & 0.02 & -0.11 & 0.13 & $-0.16^{* *}$ \\
\hline & $(0.10)$ & $(0.09)$ & $(0.10)$ & $(0.08)$ \\
\hline
\end{tabular}

Significance: *** 99\%, ** 95\%,* 90\%. Probit model with dependent variables the choices $\mathrm{A}(\mathrm{C})$ and $\mathrm{D}(\mathrm{F})$ and as regressors dummy variables for the Steps of reasoning of the IBR model. Marginal effects evaluated at the mean are reported with relative standard errors in parenthesis. 
Figure 1: Distribution of choices in the guessing game

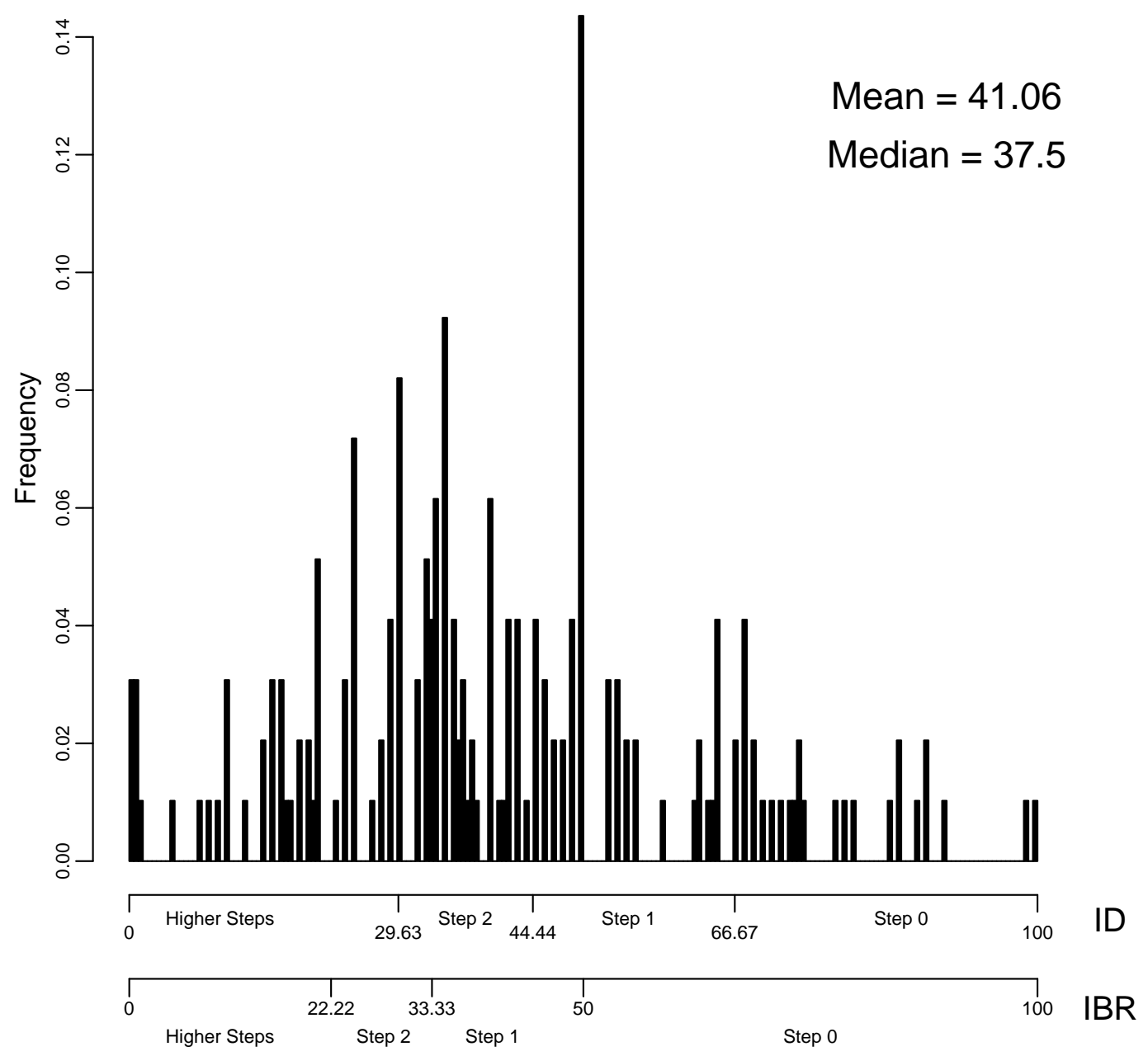

Step 1 for IBR model includes the value 50 . 
Figure 2: Fraction of subjects' choices

Equality vs Self-regard

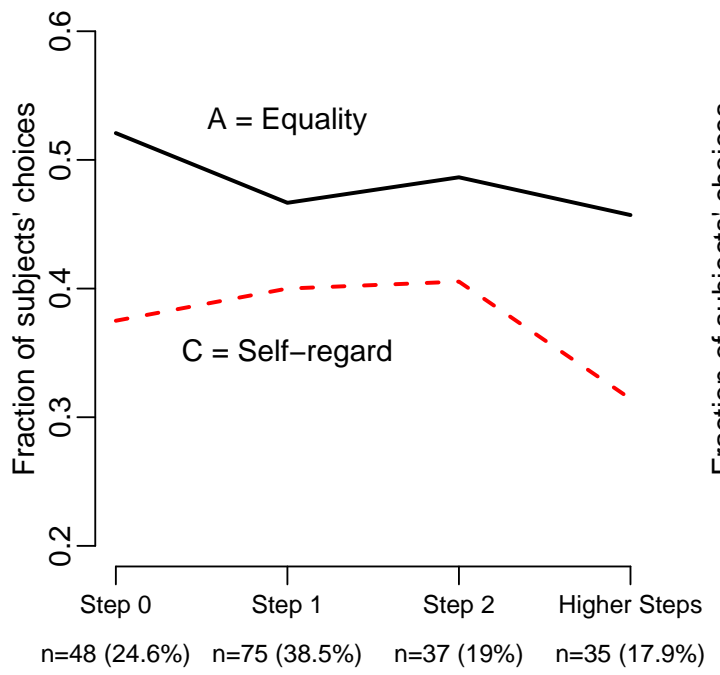

Efficiency vs Self-regard

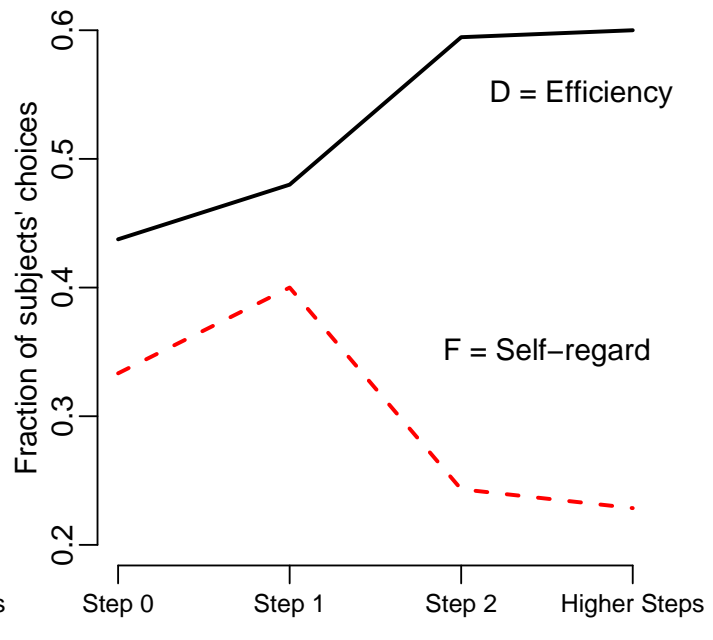

Figure 2 Fraction of subjects' choices choosing $\mathrm{A}(\mathrm{C})$ or $\mathrm{D}(\mathrm{F})$ on the total number of subjects in the same step. $n$ reports number of subjects per step and total percentage of subjects in the step in the total sample(195).

Figure 3: Mean guess divided by median guess

Equality vs Self-regard

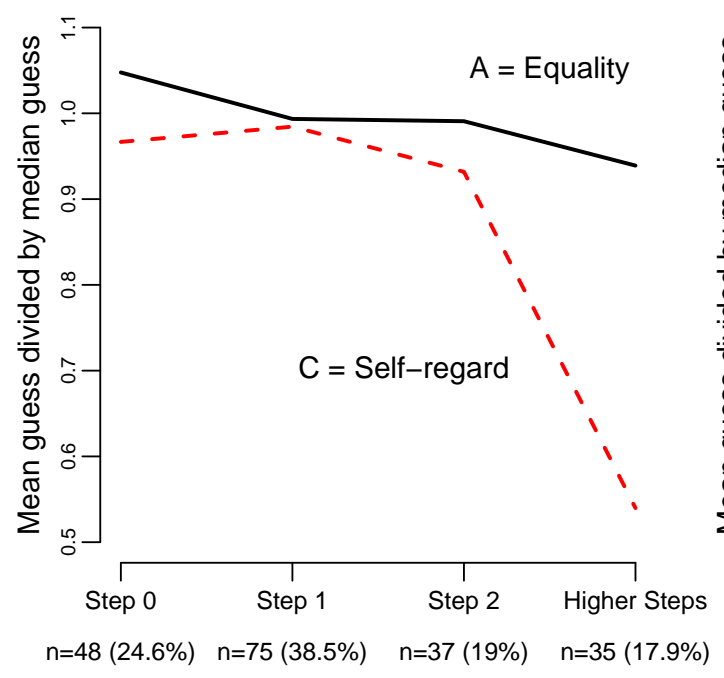

Efficiency vs Self-regard

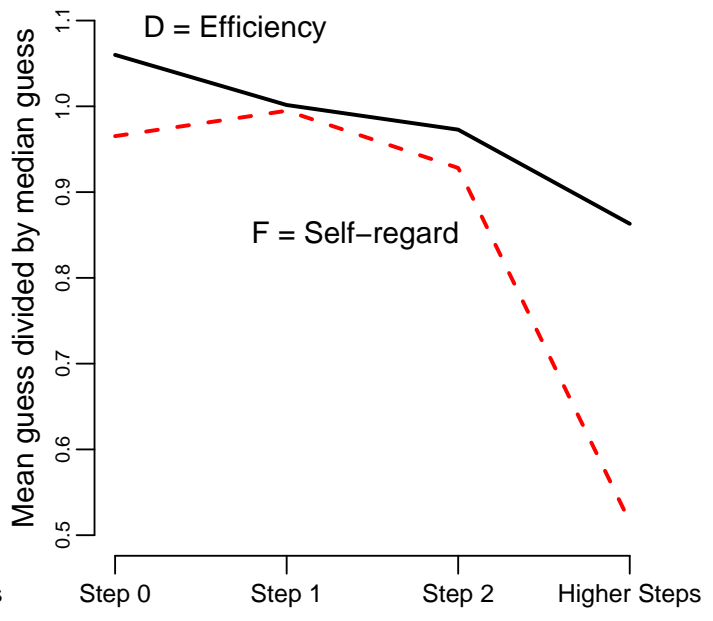

Figure 3 the mean guess of subjects choosing $\mathrm{A}(\mathrm{C})$ ( or $\mathrm{D}(\mathrm{F})$ ) divided by the median guess in the same step. 


\section{Appendix - Not for publication}

Table 4: Guess and concerns for efficiency and equality.

\begin{tabular}{|c|c|c|c|c|}
\hline & \multicolumn{4}{|c|}{ Dependent Variable } \\
\hline \multirow{3}{*}{ guess } & A (equality) & $\mathrm{C}$ (self-regard) & D (efficiency) & F (self-regard) \\
\hline & 0.001 & -0.01 & -0.001 & 0.0003 \\
\hline & $(0.001)$ & $(0.001)$ & $(0.001)$ & $(0.001)$ \\
\hline
\end{tabular}

Significance: *** 99\%, ** 95\%,* 90\%. Probit model with dependent variables the choices A(C) and $\mathrm{D}(\mathrm{F})$ and independent variables, the value of the guess of each subject, "guess". Standard errors in parenthesis.

Table 5: Steps of reasoning and concerns for efficiency and equality.

\begin{tabular}{lllll}
\hline \multicolumn{5}{l}{ Dependent Variable } \\
\multirow{2}{*}{ guess } & A (equality) & $\mathrm{C}$ (self-regard) & $\mathrm{D}$ (efficiency) & $\mathrm{F}$ (self-regard) \\
\cline { 2 - 5 } & 0.007 & $-0.01^{* * *}$ & 0.007 & $-0.01^{* *}$ \\
Step 0 & $(0.004)$ & $(0.004$ & $(0.004)$ & $(0.004)$ \\
& -0.14 & $0.34^{* *}$ & -0.24 & 0.21 \\
Step 2 & $(0.15$ & $(0.15)$ & $(0.16)$ & $(0.14)$ \\
& 0.11 & -0.17 & $0.20^{*}$ & $-0.29^{* * *}$ \\
Higher Steps & 0.19 & $(0.11)$ & $(0.11)$ & $(0.11)$ \\
& $(0.11)$ & $-0.48^{* * *}$ & $0.32^{* *}$ & $-0.48^{* * *}$ \\
& $(0.16)$ & $(0.17)$ & $(0.17)$ & $(0.16)$ \\
\hline
\end{tabular}

Significance: *** 99\%,** 95\%,* $90 \%$. Probit model with dependent variables the choices $\mathrm{A}(\mathrm{C})$ and $\mathrm{D}(\mathrm{F})$ and independent variables, the value of the guess of each subject, "guess", and dummy variables corresponding to the Steps of reasoning in the IBR model. Standard errors in parenthesis. 